(RESEARCH ARTICLE)

\title{
Comparing microbial community heterogeneity in soil under organic and conventional agricultural system cultivated with olive and peach trees in three fields differ with age at Al-Jouf Saudi Arabia
}

\author{
Ahmad Yassin Majjami 1, Abdullah Saad Al-Modaihsh 1, Fahad Nasser Al-Barakah 1, Mohamed Hamza El- \\ Saeid ${ }^{1}$, Samir Gamil Al-Solimani ${ }^{2}$ and Fahad Mohammed Alghabari 2,* \\ ${ }^{1}$ Department of Soil Sciences, College of Food and Agriculture Sciences, King Saud University, P.O. Box 2460, Riyadh \\ 11451, Kingdom of Saudi Arabia. \\ 2 Department of Arid Land Agriculture, Faculty of Meteorology, Environment and Arid Land Agriculture, King Abdul-Aziz \\ University, Jeddah, Saudi Arabia P.O. Box: 80208 Jeddah: 21589.
}

Publication history: Received on 18 September 2020; revised on 27 September 2020; accepted on 29 September 2020

Article DOI: https://doi.org/10.30574/wjarr.2020.7.3.0350

\begin{abstract}
This research study was carried out at Al-Jouf region, north eastern Saudi Arabia, to investigate the differences in microbial community and density of microorganisms in the rhizosphere of olive (Olea europaea) and peach (Prunus persica) tree fields subjected to growth under organic and conventional agriculture systems. The soil analytical processes were conducted in three olive and three peach fields $(9,17,27$ years old and 20 ha in area) under organic fertilization, and three similar olive and peach fields under conventional fertilization system. The results indicated significant variations between the two agricultural systems in rhizosphere content of microbial and mycorrhizal colonization\%. The highest percentages of actinomycetes, total fungi and bacteria (PSM) were recorded in the organically agricultural system (OAS) in olive fields in comparison to conventionally agricultural system (CAS). Regarding field ages OAS dominated CAS in total number of fungi under all olive fields ages, while under peach fields fungi counts were significantly high under CAS compared to OAS. Under peach fields OAS dominated giving the highest actinomycetes and bacteria and CAS dominated by giving the highest CFU and fungi count. The significantly highest counts of CFU and bacteria were attained under conventional farming system (CAS) in all of the different aged field compared to OAS. Actinomycetes and bacteria in young fields were significantly high under CAS, while in old fields actinomycetes and fungi counts were the highest under OAS.
\end{abstract}

Keywords: Organic agricultural system (OAS); Conventional agricultural system (CAS); Olive; peach fields; Fertilization system; Al-Jouf region.

\section{Introduction}

It can be said that till now it couldn't be assumed that organic farming systems comprise larger and more active soil microbial communities compared to conventional farming systems. Current literature concerning differences in soil microbial abundance and activity in organic and conventional cropping systems was analyzed and results indicated that differences in microbial size and activity between organic and conventional farming systems vary as a function of land use (arable, orchards, and grassland), plant life cycle (annual and perennial) and climatic zone [1]. Diversity and abundance of microbial communities in soil under organic and conventional farming systems is not fully understood, in spite of their crucial role in agro-ecosystems [2,3]. Organic farming system is more favorable and beneficial for biodiversity in microorganism, but other factors may cause variation in these effects [4]. Soil biological properties are

\footnotetext{
${ }^{*}$ Corresponding author: Fahad Mohammed Alghabari

Department of Arid Land Agriculture, Faculty of Meteorology, Environment and Arid Land Agriculture, King Abdul-Aziz University, Jeddah, Saudi Arabia P.O. Box: 80208 Jeddah: 21589.
} 
more favorable under organically managed soils than conventionally managed soils which are essential for functions in the given ecosystem [5]. Many other authors found no significant differences between conventional systems and organic management systems in decrease or abundance of bacteria [6, 7]. Weather conditions and seasonal changes must be taken into account when studying fertilization system effects, because they might affect soil biological properties, $[8,9,10]$, and $[11]$ admitted that microbial communities are more sensitive indicators of variation in soil quality than chemical and physical soil properties. Organic agricultural management systems were suggested positive in many studies $[6,12,13,14,15]$, but the effects of farming systems whether organic or conventional on the diversity of microbial communities still complicated and controversial [16] as many authors such as [12]found an increase in diversity after manure amendment.

The aim of this research is to study the difference between organic and conventional agriculture systems applied in the olive and peach tree fields at Al-Jouf region, north eastern Saudi Arabia, regarding diversity and density of microorganisms in the rhizosphere of olive and peach tree fields.

\section{Material and methods}

\subsection{Selection of fields and collection of plants' roots and soil samples}

During the April 2018, the area of Al-Jouf was surveyed and fields of olive and peach trees subjected to organic agriculture system and conventional agriculture system were chosen for study. Three farms (representing three ages, 9, 1727 years) for olive (Olea europaea) and three farms for peach (Prunus persica) were chosen for agro-ecosystems practices. Area of each farm is about 20 ha, and trees were planted at rate of 200 trees/ ha, at $(10 \mathrm{~m}$ distance between the tree rows). The trees are commonly fertilized only by organic sources. Other similar three farms of olive and three farms of peach trees were selected, from the adjacent farms in (Al-Busaita farms) representing the conventional agriculture system of fertilization where no organic fertilizers were used.

\subsection{Collection of rhizosphere soil samples}

The rhizosphere trees were collected. Counts of soil microorganisms were determined in rhizosphere soil samples. Specific media was used to measure counts of microorganisms. The serial dilution plate method or the most probable number method was used for counting total microbial counts on nutrient agar medium; total fungi count on Martin's Medium; phosphate dissolving bacteria on modified Bunt and Rovira medium [17]; and Streptomyces sp. on Jensen's medium.

\subsection{Collection of roots and rhizosphere soils samples.}

Roots and rhizosphere soils of different plants were collected from the different farms, in Three replicated. Rhizosphere soils, fine and feeder roots were collected by digging the soil with a soil corer. In the laboratory, roots were separated immediately from the soil and preserved in 50\% alcohol. Soil samples were immediately studied to avoid the damage of the spores in the rhizosphere soil. From each sample, 100g soil were taken in a bucket of 10 liters' capacity and 5 liters of water were mixed with the soil. The soil sample was mixed well with water to make a soil-water suspension. The suspension was left for five minutes for settling down of insoluble and heavy particles. The suspension was passed through the ASTM-60, ASTM-100, ASTM-240 and ASTM-400 sieves gradually to extract the spores followed by wet sieving and decanting method [18]. The residues of the sieves were filtered with the Whitman filter paper No-1. Squares of intersecting gridlines were drawn earlier on the filter paper for easy counting of spore. After water filtration the paper was examined under the stereo-binocular microscope at $2.5 \times 10$ magnification and the number was recorded. Spores were separated on the basis of morphological characters and then they were observed under compound microscope mounting on - and Melzer's reagent and observed under digital photographic microscope 'Olympus DP72' at $10 \times 0.10$ and $10 \times 0.25$ magnification. Preserved roots were washed carefully to remove the alcohol and cut into $1 \mathrm{~cm}$ length for AM fungal structural analysis. Roots were heated at $80^{\circ} \mathrm{C}$ for $20-30$ minutes in $10 \% \mathrm{KOH}$ and thereafter left overnight in $1 \% \mathrm{HCl}$. Deeply pigmented roots were treated with 3\% $\mathrm{H} 2 \mathrm{O} 2$ to remove the lignin and to clearer. Cleared roots were stained with trypan-blue following the method of [19]. 20-30 segments were mounted on a slide and examined under digital photographic microscope 'Olympus DP72' at $10 \times 0.10$ and $10 \times 0.25$ magnification. Presence of mycelium, vesicles and arbuscules were observed. Mycelial colonization was regarded as total AM colonization. Percent colonization was calculated by the following formula:

$$
\% \text { Colonization }=\frac{\text { Total No. of AM positive segments }}{\text { Total No. of segments studied }} \times 100
$$




\subsection{Statistical analysis}

Data analysis was done using Microsoft excel 2010 program.

\section{Results and discussion}

\subsection{Olive fields of the organic farms.}

\subsubsection{Total microbial counts}

The average percentages of microbial counts in the rhizosphere of olive tree fields $(9,17,27$ years old) under both organic and conventional farming systems farms are presented in table (1).

The results illustrate that the significantly highest total colony counts were recorded in the rhizosphere soil of the 27 years old olive field under conventional farming system recording an average of $18.56 \times 107 \mathrm{CFU}$ compared to $3.31 \mathrm{x} 107$ CFU in the 27 years old field soil under organically fertilized field. Seconded by the organically fertilized soil of the 17 years old field with $9.04 \times 107 \mathrm{CFU}$ compared to total count number in field soil of 17 years' age field under conventional system with an average of $0.21 \times 107 \mathrm{CFU}$.

The significantly highest total soil count of actinomycetes was attained in the rhizosphere soil of the 17 years old field under organic farming system with 5.2x105 CFU compared to 2.95x105 CFU in the same aged field under conventional fertilization system. But in the case of the actinomycetes count in soil of the younger field ( 9 years) the count was significantly high under conventional system (4.21x105 CFU) compared to organic system with (1.09x105 CFU) and $1.59 \times 105 \mathrm{CFU}$ compared to their corresponding fields under conventional system. But for the younger field (9 years old) actinomycetes.

As for total fungi count in the rhizosphere soil, the highest fungi count $6.64 \times 104$ was in the field aged 27 years under OAS compared to the 27 years old field under CAS with 5.20x104, then comes fungi soil content in the younger field

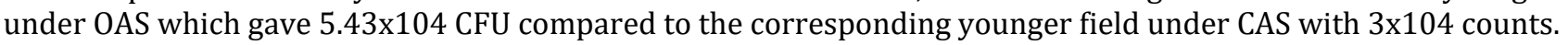

Regarding phosphate dissolving bacteria (PSM) At the rhizosphere soil of the 17 years old field under OAS dominated giving the highest bacteria number 10.04x104 compared to field 17 years under CAS with only 1.8x104 bacteria count. Then the youngest olive field under CAS attained significantly the highest PSM (6.70x104) compared to the corresponding 9 years old field under OAS with only $1.26 \times 104$.

It could be noted That the rhizosphere soil of the 17 years old field recorded the highest total microbial counts, actinomycetes and PSM under the OAS. As for the conventional fertilizing system, the old field (27 years) attained the highest average in total microbial counts and total fungi, while the younger field ( 9 years) dominated in the values of actinomycetes and PSM.

Table 1 Microbial properties of soil in organic olive fields

\begin{tabular}{|l|l|l|l|l|l|}
\hline \multirow{2}{*}{ Times } & & $\begin{array}{l}\text { Total microbial } \\
\text { count } \mathbf{X 1 0 7 )}\end{array}$ & $\begin{array}{l}\text { Actinomycetes } \\
\mathbf{( X 1 0 5 )}\end{array}$ & $\begin{array}{l}\text { Total Fungi count } \\
\mathbf{( X 1 0 4 )}\end{array}$ & $\begin{array}{l}\text { PSM } \\
(\mathbf{X 1 0 4 )}\end{array}$ \\
\hline \multirow{2}{*}{ A1 } & OAS & 0.07 & 1.09 & 5.43 & 1.26 \\
\cline { 2 - 6 } & CAS & 0.39 & 4.21 & 3.00 & 6.70 \\
\hline \multirow{2}{*}{ A2 } & OAS & 9.05 & 5.28 & 2.03 & 10.04 \\
\cline { 2 - 6 } & CAS & 0.21 & 2.95 & 3.48 & 1.80 \\
\hline \multirow{2}{*}{ A3 } & OAS & 3.31 & 1.59 & 6.64 & 2.66 \\
\cline { 2 - 6 } & CAS & 18.56 & 0.56 & 5.20 & 2.81 \\
\hline
\end{tabular}




\subsection{Peach fields of the organic farms}

\subsubsection{Total microbial counts}

Table (2) is showing averages of total microbial, actinomycetes, fungi and PSM counts in the rhizosphere of peach trees in the three fields 9, 17 and 27-year-old under organic and conventional farms. The significantly highest total colony counts were recorded in the rhizosphere soil of the 27 years old peach field under conventional farming system recording an average of $20.45 \times 107$ CFU compared to $0.99 \times 107$ CFU in the 27 years old field soil under organically fertilized field. Seconded by the organically fertilized soil of the 17 years old field with $11.50 \times 107$ CFU compared to total count number in field soil of 17 years' age field under conventional system with an average of $0.84 \times 107$ CFU.

The significantly highest total soil count of actinomycetes was attained in the rhizosphere soil of the 27 years old field under organic farming system with 6.24x105 CFU compared to 4.04x105 CFU in the same aged field under conventional fertilization system. Also in the rhizosphere of the younger field ( 9 years) the actinomycetes count was significantly high under organic fertilization system (5.34x105 CFU) compared to organic system with (2.99x105 CFU).

As for total fungi count in rhizosphere conventional fertilization system dominated organic fertilization giving the highest counts in all peach fields. The highest fungi count $5.31 \times 104$ was in the field aged 27 years under CAS compared to the 27 years old field under OAS with $3.64 \times 104$, then comes fungi soil content in the younger field under CAS which gave 4.35x104 CFU compared to the corresponding younger field under OAS with 3.47x104 counts.

Regarding phosphate dissolving bacteria (PSM) count the 27 years old field under OAS dominated giving the highest bacteria number 3.10x104 compared to field 27 years under CAS with only $1.71 \times 104$ bacteria count. Then the 17 years old peach field under CAS attained significantly the highest PSM (2.76x104) compared to the corresponding 17years old field under OAS with only 1.78x104.

It could be noted that under peach Trees the rhizosphere of the oldest field (27 years old) recorded the highest total microbial counts (under CAS), the highest actinomycetes and PSM (under the OAS). As for the total fungi count the highest counts were recorded in rhizosphere of the 17 years old field under conventional fertilizing system.

Table 2 Microbial properties of soil in organic of peach fields

\begin{tabular}{|l|l|l|l|l|l|}
\hline & & $\begin{array}{l}\text { Total Microbial } \\
\text { count (X107) }\end{array}$ & $\begin{array}{l}\text { Actinomycete } \\
\text { (X105) }\end{array}$ & $\begin{array}{l}\text { Total Fungi count } \\
\mathbf{( X 1 0 4 )}\end{array}$ & $\begin{array}{l}\text { PSM } \\
\mathbf{( X 1 0 4 )}\end{array}$ \\
\hline \multirow{2}{*}{ A1 } & $\begin{array}{l}\text { OA } \\
\text { S }\end{array}$ & 0.19 & 5.34 & 0.55 & 0.36 \\
\cline { 2 - 6 } & CAS & 2.64 & 2.99 & 3.77 & 1.50 \\
\hline \multirow{2}{*}{ A2 } & $\begin{array}{l}\text { OA } \\
\text { S }\end{array}$ & 11.50 & 1.13 & 1.64 & 1.78 \\
\cline { 2 - 6 } & CAS & 0.84 & 3.95 & 5.31 & 2.76 \\
\hline \multirow{2}{*}{ A3 } & $\begin{array}{l}\text { OA } \\
\text { S }\end{array}$ & 0.99 & 6.12 & 3.47 & 3.10 \\
\cline { 2 - 6 } & CAS & 20.45 & 4.04 & 4.35 & 1.71 \\
\hline
\end{tabular}

\subsection{Mycorrhiza colonization percentage}

The total mycorrhizal colonization percentages were determined in the different field rhizosphres in olive and peach fields under both OAS and CAS.

The results in table (3) illustrate that organically fertilized 17 years old peach field recorded the highest averages in total rhizosphere colonization percentages registering values of $(85.25,56.75,23.25 \%$ and 446.75 spore count/100g dry soil) respectively compared to averages of these parameters in the soils of the 17 years old fields under CAS. But CAS dominated OAS in all of these parameters in the old fields (27 years old) root rhizosphere recording significantly the highest averages in percentages of mycelium, vesicles, arbuscules, spore count $(55,23.5,30 \%$ and 79.25 spore) 
respectively compared to the 27 years old field under OAS which recorded $(23.25,13.5,8.5 \%$ and 17.5 spores) respectively. So OAS dominated CAS in field 17 years old, and CAS dominated OAS in field 27 years old.

Table 3 Mycorrhizal colonization\% in soil in conventional olive fields

\begin{tabular}{|l|l|l|l|l|l|}
\hline \multirow{2}{*}{ Times } & \multicolumn{4}{|l|}{ Total colonization (\%) } & $\begin{array}{l}\text { Spore Count/100g } \\
\text { dry soil }\end{array}$ \\
\cline { 2 - 6 } & & Mycelium & Vesicles & Arbuscules & \\
\hline \multirow{3}{*}{ A1 } & OAS & 56.50 & 36.75 & 13.50 & 42.50 \\
\cline { 2 - 6 } & CAS & 61.50 & 23.50 & 31.75 & 22.50 \\
\hline \multirow{3}{*}{ A2 } & OAS & 85.25 & 56.75 & 23.25 & 446.75 \\
\cline { 2 - 6 } & CAS & 78.25 & 53.50 & 20.00 & 151.00 \\
\hline \multirow{2}{*}{ A3 } & OAS & 23.25 & 13.50 & 8.50 & 17.50 \\
\cline { 2 - 6 } & CAS & 55.00 & 23.50 & 30.00 & 79.25 \\
\hline
\end{tabular}

\subsection{Peach Fields}

The results in table (4) illustrate that organically fertilized 17 years old peach field recorded the highest averages in total rhizosphere colonization percentages registering values of $(78.50,38.350,18.25 \%$ and 237.25 spore count $/ 100 \mathrm{~g}$ dry soil) respectively compared to averages of these parameters in the soils of the 17 years old fields under CAS. But CAS dominated OAS in all of these parameters in the youngest ( 9 years) and the old (27 years) fields root rhizosphere recording significantly the highest averages in percentages of mycelium, vesicles, arbuscules, spore count than OAS. So OAS dominated CAS in field 17 years old, and CAS dominated OAS in fields 9 and 27 years old.

Table 4 Mycorrhizal colonization in soil of organic peach field

\begin{tabular}{|l|l|l|l|l|l|}
\hline \multirow{2}{*}{ Times } & & \multicolumn{3}{|l|}{ Total colonization (\%) } & \multirow{2}{*}{ Spore Count/100g dry soil } \\
\cline { 3 - 6 } & & Mycelium & Vesicles & Arbuscules & \\
\hline \multirow{2}{*}{ A1 } & OAS & 31.50 & 11.75 & 3.25 & 29.00 \\
\cline { 2 - 6 } & CAS & 45.25 & 25.00 & 5.00 & 51.25 \\
\hline \multirow{3}{*}{ A2 } & OAS & 78.25 & 38.50 & 18.25 & 237.25 \\
\cline { 2 - 6 } & CAS & 50.00 & 23.25 & 8.25 & 120.00 \\
\hline \multirow{2}{*}{ A3 } & OAS & 43.25 & 10.00 & 13.50 & 43.25 \\
\cline { 2 - 6 } & CAS & 61.50 & 37.00 & 14.75 & 58.00 \\
\hline
\end{tabular}

\section{Discussion}

[20] Reported a difficulty in assessing differences between organic and conventional agriculture systems in soil physical, chemical and biological characteristics due to variation in soil type, crops, climatic conditions, type of amendments and the amount applied.

The results obtained in this study indicated significant variations between the two agricultural systems applied in the fields of olive and peach trees at Al-Jouf region north east of the Kingdom of Saudi Arabia regarding trees root rhizosphere content of microbial and mycorrhizal colonization\%. The organically agricultural system (OAS) in olive fields recorded the highest percentages in actinomycetes, total fungi and in phosphate dissolving bacteria (PSM) in comparison to conventionally agricultural system (CAS), and the CAS dominated the OAS by giving the highest total microbial count (CFU). The results obtained in this study on olive fields agree with that obtained by [5] in India, who found significant increase in population of bacteria, fungi and actinomycetes by $56.9,55.2$ and $49.5 \%$, respectively at all the locations under organic farming when compared to those in the conventionally managed soils. [21] assumed that the higher microbial population in organically fertilized soils may be due to a higher organic matter content, water 
retention characteristics and nutrient availability. In peach field tree roots rhizosphere (OAS) recorded the highest percentages in actinomycetes and in phosphate dissolving bacteria (PSM) while CAS dominated the OAS by giving the highest total microbial count ( $\mathrm{CFU}$ ) and total fungi count. It is noted that under olive fields total fungi count was significantly high under OAS in all field ages compared to CAS, while under peach fields fungi counts were significantly high in all field ages under CAS compared to OAS. As can be seen that in peach fields both OAS and CAS dominated (OAS gave the highest actinomycetes and bacteria) and CAS gave the highest CFU and fungi. This might be due to variation in soil texture and structure. and this disagrees with results of [22] who found no differences between conventional and organic agriculture systems on soil microorganism populations (fungus, bacterium and actinomycetes), for the corn (Zea mays) and tomato (Lycopersicum esculentum).

According to field age, tree roots rhizosphere content of the younger field ( 9 years) olive trees under CAS dominated in the highest counts of $\mathrm{CFU}$, actinomycete and bacteria, and also in the youngest peach fields giving the highest counts of CFU, fungi and bacteria counts compared to OAS. In 17 years old field OAS dominated CAS giving the highest counts in total microbial count (CFU), actinomycete and bacteria count. For the old field (27 years) of olive CAS dominated giving the highest counts of CFU and bacteria counts, while OAS dominated in counts of actinomycetes and fungi, and in peach fields CAS dominated giving the highest counts of CFU, actinomycetes and fungi, while OAS only dominating in bacteria. So it is clear that in young fields actinomycetes and bacteria were high under CAS, while in old fields actinomycetes and fungi counts were the highest under OAS, and this may indicate that by time more organic nutrients may accumulate in the soil making it favorable for growth of fungi. This agrees with results of [23] in Egypt who concluded that microbial communities (bacteria, actinomycetes and fungi) were greater in soils from organic system compared to conventional system and increase by time.

Regarding microhyzal colonization, they have an important role in plant nutrition, mycorrhizas can influence soil aggregation, and arbuscular mycorrhizal fungi (AMF) play a crucial role in nutrient acquisition and soil fertility [24]. The results obtained showed that the (OAS) in olive fields recorded the highest percentages in myceliam, vesicles and in spore counts $/ 100 \mathrm{~g}$ dry soil, in comparison to conventionally agricultural system (CAS), while CAS dominated the OAS only in arbuscule percentage. And in peach field tree roots rhizosphere under (OAS) recorded the highest percentages in all these four parameters mycelium, vesicles, arbuscule and in spore counts/100 g dry soil comparison to (CAS). These results are incompatible with the results of [25] who reported the natural presence of arbuscular mycorrhizal fungi species in organic farming ecosystems and their severe depression under conventional farming, indicating a potentially severe loss of ecosystem function under conventional farming.

Regarding field age, in the younger field ( 9 years) OAS dominated giving the highest vesicles percentage and spore counts $/ 100 \mathrm{~g}$ dry soil in olive trees rhizosphere compared to CAS, while CAS dominated in the mycelium and arbuscules percentages compared to OAS. In the 17 years old field in olive OAS gave significantly the highest values of all the four parameters compared to CAS. While at the same time CAS dominated OAS in the oldest field (27 years) under olive trees recording the highest percentages and counts in all of the studied parameters. This means that when olive fields are young organic agricultural system develops significantly high percentages of mycelium, vesicles, arbuscules and spore count/100 dry soil compared to conventionally agricultural system, but under old olive fields conventional agricultural system dominates in all these studied parameters compared to OAS. In case of peach fields CAS dominated OAS in the younger ( 9 years), and 27 years old field in all of the four studied parameters giving the highest percentages of mycelium, vesicles, arbuscules and spore count $/ 100 \mathrm{~g}$ dry soil, while OAS dominated CAS in the 17 years old field giving the highest rhizosphere contents of mycelium, vesicles, arbuscules and spore count/100g dry soil. This indicates that under olive or peach the 17 years old field under OAS dominates CAS giving the highest values of the studied parameters, while under the old field 27 years old field CAS dominates OAS in all these studied parameters.

\section{Conclusion}

The results indicated significant variations between the two agricultural systems applied in the fields of olive and peach trees at Al-Jouf region north east of the Kingdom of Saudi Arabia regarding rhizosphere content of microbial and mycorrhizal colonization\%. The highest percentages of actinomycetes, total fungi and bacteria (PSM) were recorded in the organically agricultural system (OAS) in olive fields in comparison to conventionally agricultural system (CAS). Regarding field ages OAS dominated CAS in total number of fungi under all olive fields ages, while under peach fields fungi counts were high under CAS compared to OAS. Under peach fields OAS dominated (giving the highest actinomycetes and bacteria and CAS dominated by giving the highest CFU and fungi count. The significantly highest counts of CFU and bacteria were attained under conventional farming system (CAS) in all the different aged field compared to OAS. Actinomycetes and bacteria in young fields were significantly high under CAS, while in old fields actinomycetes and fungi counts were the highest under OAS, and this may indicate that by time more organic nutrients may accumulate in the soil making it favorable for growth of fungi. OAS recorded the highest percentages in myceliam, 
arbuscules, vesicles and in spore counts/100g dry soil in both olive and peach fields in comparison to conventionally agricultural system (CAS), except arbuscule counts under olive. Regarding field age OAS gave significantly the highest values of all studied four parameters (myceliam, arbuscules, vesicles and in spore counts/100g dry soil) in the 17 years old olive field compared to CAS. CAS dominated in the 27 years old field under olive trees recording the highest percentages and counts in all of the studied parameters.

\section{Compliance with ethical standards}

\section{Acknowledgments}

The authors would like to acknowledge the immense contribution laboratory staff of department of soil sciences, College of Food and Agriculture Sciences, King Saud University for their technical assistance.

\section{Disclosure of conflict of interest}

The authors declare that they have no conflict of interest.

\section{References}

[1] Lori M, Symnaczik S, Mäder P, De Deyn G, Gattinger A. Organic farming enhances soil microbial abundance and activity-A meta-analysis and meta-regression. PLoS On. 2017; 12(7): e0180442.

[2] Melero S, Porras JCR, Herencia JF, Madejon E. Chemical and biochemical properties in a silty loam soil under conventional and organic management, , Soil till. Res. 2006; 90: 162-170.

[3] Araujo ASF de, Santos VB, Monteiro RTR. Responses of soil microbial biomass and activity for practices of organic and conventional farming systems in Piauí state, Brazil. European Journal of Soil Biology. 2008; 44(2): 225-230.

[4] Bengtsson J, Ahnstrom J, Weibull A. The effects of organic agriculture on biodiversity and abundance: a metaanalysis. Journal of applied ecology. 2005; 42(2): 261-269.

[5] Singh SH, Phogat VK, Dahiya R, Dhull S, Kakar R. Comparative effect of organic and conventional farming practices on micronutrient content in different textured soils of Haryana, India. Int. J. Curr. Microbial. App. Sci. 2018; 7: 3399-3407.

[6] Liu B, Tu C, Hu SJ, Gumpertz M, Ristaino JB. Effect of organic, sustainable, and conventional management strategies in grower fields on soil physical, chemical, and biological factors and the incidence of Southern blight. Appl. Soil Ecol. 2007; 37: 202-214.

[7] Reilly K, Cullen E, Lola-Luz T, Stone D, Valverde J, Gaffney M, et al. Effect of organic, conventional and mixed cultivation practices on soil microbial community structure and nematode abundance in a cultivated onion crop. J. Sci. Food Agric. 2013; 93: 3700-3709.

[8] Bastida F, et al. Past, present and future of soil quality indices: a biological perspective. Geoderma. 2008; 147(34): 159-171.

[9] Meier C, Wehrli B, Van der Meer J. Seasonal fluctuations of bacterial community diversity in agricultural soil and experimental validation by laboratory disturbance experiments. Microbial Ecology. 2008; 56: 210-22.

[10] Doran JW, Elliott ET, Paustian K. Soil microbial activity, nitrogen cycling, and long-term changes in organic carbon pools as related to fallow tillage management. Soil Till Res. 1998; 49: 3-18.

[11] Turco RF, Kennedy AC, Jawson MD. Microbial indicators of soil quality. In: SSSA. 1994; 35: 73-90.

[12] Zhang Ge Y, Zhang JB, Yang LM, He JZ. Long-term fertilization regimes affect bacterial community structure and diversity of an agricultural soil in northern China. J. Soils Sediments. 2008; 8: 43-50.

[13] Jonason D, Andersson GKS, Ockinger E, Rundlof M, Smith HG, Bengtsson J. Assessing the effect of the time since transition to organic farming on plants and butterflies. J. Appl. Ecol. 2011; 48: 543-550.

[14] Chaudhry V, et al. Changes in bacterial community structure of agricultural land due to long-term organic and chemical amendments. Microbial ecology. 2012; 64(2): 450-460.

[15] Hartmann M, Frey B, Mayer J, Mader P, Widmer F. Distinct soil microbial diversity under long term organic and conventional farming. ISME Journal. 2014; 210. 
[16] Kleijn D, Berendse F, Smit R, Gilissen N. Agri-environment schemes do not effectively protect biodiversity in Dutch agricultural landscapes. Nature. 2014; 413: 723-725.

[17] Abdel-Hafez AM. Some studies on acid producing microorganisms in soil and rhizosphere with special reference to phosphate dissolvers. PhD Thesis.

[18] Phillips JM, Hayman DS. Improved procedures for clearing roots and staining parasitic and vesicular-arbuscular mycorrhizal fungi for rapid assessment of infection. Transactions of the British mycological Society. 1970; 55(1): 158-161.

[19] Herencia JF, Ruiz JC, Melero S, Galavis PG, Maqueda C. A short-term comparison of organic v. conventional agriculture in a silty loam soil using two organic amendments. The Journal of Agricultural Science. 2018; 146(6): 677.

[20] Gerdemann JW, Nicolson TH. Spore of mycorrhizal endogone species extracted from soil by wet sieving and decanting trans Br. Mycol. Soc. 1963; 46: 235-244.

[21] Grayston S, CamPbell C, Bardgett R, Mawdsley J, Clegg C, Ritz K. Assessing shifts in microbial community structure across a range of grasslands of differing management intensity using CLPP, PLFA and community DNA techniques. Applied Soil Ecology. 2004; 25: 63-84.

[22] Bettiol W, Ghini R, Galvão JAH, Ligo MAV, Mineiro JLDC. Soil organisms in organic and conventional cropping systems. Scientia Agricola. 2002; 59(3): 565-572.

[23] Daif MA, et al. Chemical and biological indicators of soil quality in organic and conventional farming systems. Journal of Soil Sciences and Agricultural Engineering. 2013; 4(11): 1273-1288.

[24] Rillig MC, Mummey DL. Mycorrhizas and soil structure. New Phytol. 2006; 171: 41-53.

[25] Oehl F, Sieverding E, Mäder P, Dubois D, Ineichen K, Boller T, Wiemken A. Impact of long-term conventional and organic farming on the diversity of arbuscular mycorrhizal fungi. Oecologia. 2004; 138: 574-583. 